\title{
Plasma vitamin A and beta-carotene in retinitis pigmentosa
}

\author{
WAGIHA H. MASSOUD,* A. G. BIRD, AND E. S. PERKINS \\ From the Departments of Experimental Ophthalmology and Clinical Ophthalmology, \\ Institute of Ophthalmology, University of London
}

The pathogenesis of retinitis pigmentosa as an isolated genetically determined disorder is still obscure. Whether the retinopathy is due to a systemic disorder or to an abnormality restricted to the retina is uncertain. Whilst pigmentary retinopathy has been described with multiple system diseases (Laurence and Moon, 1866; Flynn and Aird, 1965; Kearns, 1965) and progressive neurological disease (Winkelman, 1932; Jampel, Okazaki, and Bernstein, 1961 ; Weiner, Konismark, Stoll, and Magladery, 1967), systemic biochemical disorders have been identified in only two diseases in which pigmentary retinopathy occurs: Refsum's syndrome (Refsum, Salmonsen, and Skatvedt, I949; Billings, O'Callaghan, and O'Day 1957; Levy, 1970) and a-betalipoproteinaemia (Bassen and Kornzweig, 1950).

Particular attention has been paid to the possible role of abnormal vitamin A metabolism because of its importance in the formation of rhodopsin. Deficiency of this vitamin in man causes retinal degeneration which can be reversed by vitamin A administration (McLaren, 1963; Norden and Stigmar, 1969; Fells and Bors, 1969, 1971), and which histologically resembles genetically determined retinitis pigmentosa (Cogan, 1950). Similarly, experimental vitamin A deprivation of rats causes degeneration of rod outer segments and finally receptor cell death. Furthermore, the pigmentary retinopathy of a-betalipoproteinaemia appears to be due to abnormalities of vitamin A metabolism (Lloyd, 1968; Sperling, Hiles, and Kennerdell, 1972).

However, therapeutic trials with vitamin A in patients with genetically determined isolated retinitis pigmentosa have given no clear indication of the role of abnormal vitamin A metabolism in the pathogenesis of this condition. The results of treatment with vitamin A appear to have impressed some investigators favourably (Town, 195I ; Friede, 1952) while others have reported little therapeutic success (Levine, I933). In I962, an uncontrolled therapeutic

Address for reprints: A. C. Bird, F.R.C.S., Department of Clinical Ophthalmology, Moorfields Eye Hospital, City Road, London EC1V 2PD

* Present address: 10, Dr. Handoussa Street, Garden City, Cairo, Egypt trial suggested that patients with retinitis pigmentosa benefited from intramuscular and oral administration of I I-cis vitamin A, but a rigidly controlled trial by the same investigators failed to confirm this (Chatzinoff, Nelson, Stahl, and Clahane, 1968). The experience of this group illustrates the difficulties in assessing the results of uncontrolled therapeutic trials, and puts into perspective the results of previous studies on the therapeutic value of vitamin $A$ in retinitis pigmentosa.

The results of metabolic studies have been similarly inconsistent. Campbell (1962), Campbell and Tonks (1962), and Campbell, Harrison, and Tonks (1964) reported reduced plasma levels of vitamin A in patients with retinitis pigmentosa when compared with levels in the general population, and concluded that vitamin A deficiency played a part in the pathogenesis of retinitis pigmentosa. They received support from some investigators (Shearer, 1964; Rogers, 1966; Soliman, Abboud, Osman, and Massoud, 1970), though others could not confirm the original findings (Mehra and Khare, 1965).

It should be emphasized that the workers who studied vitamin A metabolism and conducted therapeutic trials took no account of the mode of inheritance of retinitis pigmentosa in the patients studied. Since each form of retinitis pigmentosa is a distinct disease likely to have a different pathogenesis from other forms, mixed populations were studied. For this reason it is difficult to draw any definite conclusions from the data obtained by these workers.

It was the purpose of the present study to measure the plasma levels of vitamin A and of its precursor, beta-carotene, in patients with genetically determined retinitis pigmentosa.

\section{Material}

39 patients with genetically determined isolated retinitis pigmentosa were included in the study and the clinical details are shown in Table I.

In twelve patients the retinopathy was transmitted as an autosomal recessive characteristic (Cases $1-12$ ), in 
Table I Results in 45 patients

\begin{tabular}{|c|c|c|c|c|c|c|c|c|c|c|c|}
\hline \multirow[b]{2}{*}{$\begin{array}{l}\text { Case } \\
\text { no. }\end{array}$} & \multirow{2}{*}{$\begin{array}{l}\text { Age } \\
(y r s)\end{array}$} & \multirow{2}{*}{ Sex } & \multirow{2}{*}{$\begin{array}{l}\text { Type of } \\
\text { inheritance }\end{array}$} & \multicolumn{2}{|c|}{ Visual acuity } & \multicolumn{2}{|l|}{$E R G$} & \multicolumn{2}{|l|}{$\begin{array}{l}\text { Dark adaptation } \\
\text { (Log. units } \\
\text { above threshold) }\end{array}$} & \multirow{2}{*}{$\begin{array}{l}\text { Vit. A. } \\
\text { ( } \mu \text { g. per cent.) }\end{array}$} & \multirow{2}{*}{$\begin{array}{l}\text { beta-carotene } \\
\text { ( } \mu \text { g. per cent. })\end{array}$} \\
\hline & & & & $R$ & $L$ & $R$ & $L$ & $R$ & $L$ & & \\
\hline $\begin{array}{l}1 \\
2\end{array}$ & $\begin{array}{l}25 \\
10\end{array}$ & $\begin{array}{l}\mathrm{F} \\
\mathrm{M}\end{array}$ & $\begin{array}{l}\mathrm{AR} \\
\mathrm{AR}\end{array}$ & $\begin{array}{l}\mathrm{HM} \\
6 / 18\end{array}$ & $\begin{array}{l}6 / 36 \\
6 / 18\end{array}$ & $\overline{\text { Absent }}$ & Absent & $\begin{array}{l}- \\
(1969) 2 \cdot 5 \\
(1970) 3\end{array}$ & $\begin{array}{l}\overline{3} \\
3\end{array}$ & $\begin{array}{l}73 \\
46\end{array}$ & $\begin{array}{r}118 \\
75\end{array}$ \\
\hline $\begin{array}{l}3 \\
4 \\
5 \\
6\end{array}$ & $\begin{array}{r}11 \\
9 \\
44 \\
42\end{array}$ & $\begin{array}{l}\mathbf{M} \\
\mathbf{M} \\
\mathbf{M} \\
\mathbf{M}\end{array}$ & $\begin{array}{l}\text { AR } \\
\text { AR } \\
\text { AR } \\
\text { AR }\end{array}$ & $\begin{array}{l}6 / 60 \\
1 / 60 \\
\mathrm{PL} \\
6 / 12\end{array}$ & $\begin{array}{l}3 / 60 \\
C F \\
P L \\
6 / 12\end{array}$ & $\begin{array}{l}\text { Absent } \\
\text { Absent } \\
\text { Absent } \\
\mathrm{a}-20 \mu \mathrm{v} \\
\mathrm{b}-30 \mu \mathrm{v} \\
\quad \text { No fli }\end{array}$ & $\begin{array}{l}\text { Absent } \\
\text { Absent } \\
\text { Absent } \\
\mathrm{a}-40 \mu \mathrm{v} \\
\mathrm{b}-60 \mu \mathrm{v} \\
\text { licker }\end{array}$ & $\begin{array}{l}- \\
\overline{-} \\
\overline{3}\end{array}$ & $\begin{array}{l}3 \\
\overline{-} \\
\overline{1}\end{array}$ & $\begin{array}{l}61 \\
64 \\
60 \\
54\end{array}$ & $\begin{array}{r}77 \\
65 \\
41 \\
162\end{array}$ \\
\hline $\begin{array}{l}7 \\
8\end{array}$ & $\begin{array}{l}26 \\
44\end{array}$ & $\frac{M}{F}$ & $\begin{array}{l}\mathrm{AR} \\
\mathrm{AR}\end{array}$ & $\begin{array}{l}1 / 60 \\
6 / 12\end{array}$ & $\begin{array}{l}1 / 60 \\
6 / 9\end{array}$ & $\begin{array}{l}- \\
\mathrm{a}-40 \mu \mathrm{v} \\
\mathrm{b}-80 \mu \mathrm{v}\end{array}$ & $\begin{array}{l}\overline{\mathrm{c}} \mathrm{er} \\
\mathrm{a}-40 \mu \mathrm{v} \\
\mathrm{b}-80 \mu \mathrm{v}\end{array}$ & $\stackrel{4}{N}$ & $\stackrel{4}{N}$ & $\begin{array}{l}75 \\
61\end{array}$ & $\begin{array}{l}101 \\
142\end{array}$ \\
\hline $\begin{array}{r}9 \\
10 \\
11 \\
12\end{array}$ & $\begin{array}{r}38 \\
59 \\
40 \\
9\end{array}$ & $\begin{array}{l}\text { F } \\
\text { F } \\
\text { M }\end{array}$ & $\begin{array}{l}\text { AR } \\
\text { AR } \\
\text { AR } \\
\text { AR }\end{array}$ & $\begin{array}{l}6 / 60 \\
\mathrm{PL} \\
6 / 12 \\
6 / 60 \\
\end{array}$ & $\begin{array}{l}6 / 60 \\
\mathrm{PL} \\
6 / 24 \\
6 / 60 \\
\end{array}$ & $\begin{array}{l}\text { Absent } \\
\text { Absent } \\
\text { Absent } \\
\end{array}$ & $\begin{array}{l}\text { Absent } \\
\text { Absent } \\
\text { Absent } \\
\end{array}$ & $\begin{array}{l}\frac{3}{2} \\
-\end{array}$ & $\begin{array}{l}\frac{3}{2} \\
-\end{array}$ & $\begin{array}{l}75 \\
66 \\
59 \\
56 \\
\end{array}$ & $\begin{array}{r}107 \\
197 \\
60 \\
129 \\
\end{array}$ \\
\hline 13 & 38 & $\bar{M}$ & $\overline{\mathrm{AD}}$ & $6 / 5$ & $6 / 5$ & $\begin{array}{l}\mathrm{a}-20 \mu \mathrm{v} \\
\mathrm{b}-24 \mu \mathrm{v}\end{array}$ & $\begin{array}{l}\mathrm{a}-16 \mu \mathrm{v} \\
\mathrm{b}-24 \mu \mathrm{v}\end{array}$ & 1 & 1 & 100 & 86 \\
\hline $\begin{array}{l}14 \\
15\end{array}$ & $\begin{array}{l}41 \\
21\end{array}$ & $\stackrel{\mathrm{M}}{\mathrm{F}}$ & $\underset{A D}{A D}$ & $\begin{array}{l}6 / 24 \mathrm{pt} \\
3 / 60\end{array}$ & $\begin{array}{l}6 / 60 \\
6 / 9\end{array}$ & $\begin{array}{l}\text { Absent } \\
\mathrm{a}-40 \mu \mathrm{v} \\
\mathrm{b}-60 \mu \mathrm{v}\end{array}$ & $\begin{array}{l}\text { Absent } \\
\mathrm{a}-20 \mu \mathrm{v} \\
\mathrm{b}-20 \mu \mathrm{v}\end{array}$ & $\begin{array}{l}4 \\
4\end{array}$ & 3 & $\begin{array}{l}64 \\
66\end{array}$ & $\begin{array}{l}132 \\
113\end{array}$ \\
\hline 16 & 24 & $\mathrm{~F}$ & $\mathrm{AD}$ & $6 / 6 \mathrm{pt}$ & $6 / 9$ & $\begin{array}{l}\mathrm{a}-10 \mu \mathrm{v} \\
\mathrm{b}-20 \mu \mathrm{v}\end{array}$ & $\mathrm{a}-10 \mu \mathrm{v}$ & 0 & 1 & 64 & 169 \\
\hline $\begin{array}{l}17 \\
18 \\
19 \\
20 \\
21 \\
22 \\
23 \\
24 \\
25 \\
26\end{array}$ & $\begin{array}{r}40 \\
11 \\
6 \\
20 \\
23 \\
8 \\
6 \\
68 \\
28 \\
27 \\
38\end{array}$ & $\begin{array}{l}\text { F } \\
\text { F } \\
\text { F } \\
\text { F } \\
\text { F } \\
\text { F } \\
\text { M } \\
\text { F } \\
\text { F }\end{array}$ & $\begin{array}{l}\mathrm{AD} \\
\mathrm{AD} \\
\mathrm{AD} \\
\mathrm{AD} \\
\mathrm{AD} \\
\mathrm{AD} \\
\mathrm{AD} \\
\mathrm{AD} \\
\mathrm{AD} \\
\mathrm{AD}\end{array}$ & $\begin{array}{l}6 / 18 \\
6 / 9 \\
6 / 9 \\
6 / 18 \\
6 / 9 \\
- \\
- \\
6 / 5 \\
6 / 36 \\
-\end{array}$ & $\begin{array}{l}6 / 18 \\
6 / 9 \\
6 / 9 \\
6 / 18 \\
6 / 12 \\
- \\
6 / 5 \\
6 / 60 \\
-\end{array}$ & $\begin{array}{l}\text { Absent } \\
\overline{-} \\
\bar{A} \text { Absent } \\
\overline{-} \\
\overline{-} \\
\overline{\text { Absent }} \\
\mathrm{a}-20 \mu \mathrm{v} \\
\mathrm{b}-30 \mu \mathrm{v}\end{array}$ & $\begin{array}{l}\text { Absent } \\
\text { Absent } \\
\text { Absent } \\
\text { Absent } \\
- \\
\overline{-} \\
\overline{-} \\
\text { Absent } \\
\text { a-40 } \mu \mathrm{v} \\
\mathrm{b}-100 \mu \mathrm{v}\end{array}$ & $\begin{array}{l}3 \\
2 \cdot 5 \\
2 \\
3 \\
- \\
2 \\
0.5(40 \mathrm{~min} .) \\
1 \\
4 \\
\mathrm{~N}\end{array}$ & $\begin{array}{l}3 \\
2 \\
1 \cdot 5 \\
3 \\
-1 \cdot 5 \\
1 \cdot 5 \\
0.75 \\
1 \cdot 25 \\
3 \\
\mathrm{~N}\end{array}$ & $\begin{array}{l}71 \\
51 \\
36 \\
56 \\
64 \\
51 \\
56 \\
84 \\
69 \\
53\end{array}$ & $\begin{array}{r}77 \\
92 \\
77 \\
110 \\
109 \\
71 \\
86 \\
38 \\
52 \\
108\end{array}$ \\
\hline $\begin{array}{l}27 \\
28\end{array}$ & $\begin{array}{l}32 \\
33\end{array}$ & $\mathrm{M}$ & $\begin{array}{l}\mathrm{AD} \\
\mathrm{AD}\end{array}$ & $\begin{array}{l}2 / 60 \\
6 / 24 \\
\end{array}$ & $\begin{array}{l}6 / 24 \\
6 / 12 \\
\end{array}$ & Absent & Absent & 3 & 3 & $\begin{array}{l}48 \\
61 \\
\end{array}$ & $\begin{array}{r}175 \\
58 \\
\end{array}$ \\
\hline $\begin{array}{l}29 \\
30 \\
31 \\
\end{array}$ & $\begin{array}{l}15 \\
15 \\
19 \\
\end{array}$ & $\begin{array}{l}\mathrm{M} \\
\mathrm{F} \\
\mathrm{F} \\
\end{array}$ & $\begin{array}{l}\mathrm{S} \\
\mathrm{S} \\
\mathrm{S}\end{array}$ & $\begin{array}{l}6 / 12 \\
6 / 6 \\
6 / 60 \\
\end{array}$ & $\begin{array}{l}6 / 12 \\
6 / 9 \\
3 / 60 \\
\end{array}$ & $\begin{array}{l}\text { Absent } \\
\text { Absent } \\
\text { Absent } \\
\end{array}$ & $\begin{array}{c}\text { Absent } \\
\text { Absent } \\
\text { Absent }\end{array}$ & $\begin{array}{l}2 \\
\mathrm{~N} \\
\end{array}$ & $\begin{array}{l}2 \\
\overline{0} \cdot 5 \\
\end{array}$ & $\begin{array}{l}48 \\
50 \\
59\end{array}$ & $\begin{array}{l}98 \\
57 \\
56\end{array}$ \\
\hline 32 & $\overline{25}$ & $\overline{\mathrm{M}}$ & $\overline{X-L}$ & $6 / 36$ & $3 / 36$ & $\begin{array}{l}\overline{\mathrm{a}-3.3 \mu \mathrm{v}} \\
\mathrm{b}-3 \cdot 3 \mu \mathrm{v} \\
\text { averaged }\end{array}$ & Absent & 1 & 4 & 47 & 113 \\
\hline 33 & 21 & $\mathrm{~F}$ & $X-L$ & $6 / 12$ & $6 / 12$ & $\begin{array}{l}1 \text { st visit } \\
\mathrm{a}-15 \mu \mathrm{v} \\
\mathrm{b}-60 \mu \mathrm{v} \\
\text { averaged } \\
2 n d \text { visit } \\
\text { Absent }\end{array}$ & Absent & $\begin{array}{l}\frac{1 s t \text { visit }}{0 \cdot 75} \\
\frac{2 n d \text { visit }}{4}\end{array}$ & $0 \cdot 75$ & 45 & 160 \\
\hline $\begin{array}{l}34 \\
35\end{array}$ & $\begin{array}{l}19 \\
16\end{array}$ & $\begin{array}{l}\mathrm{M} \\
\mathbf{M}\end{array}$ & $\begin{array}{l}X-L \\
X-L\end{array}$ & $\begin{array}{l}6 / 24 \\
6 / 18\end{array}$ & $\begin{array}{l}3 / 60 \\
6 / 12 \mathrm{pt}\end{array}$ & $\begin{array}{l}\text { Absent } \\
\text { averaged }\end{array}$ & Absent & 4 st visit & 4 & $\begin{array}{l}69 \\
74\end{array}$ & $\begin{array}{r}113 \\
81\end{array}$ \\
\hline & 17 & & & & & $\begin{array}{l}5 \mu \mathrm{v} \\
\text { (2nd visit) }\end{array}$ & & $\begin{array}{l}1 \\
2 \cdot 5 d v i s i t \\
\end{array}$ & $\begin{array}{l}0.75 \\
2.5 \\
\end{array}$ & & \\
\hline $\begin{array}{l}36 \\
37\end{array}$ & $\begin{array}{l}13 \\
16 \\
\end{array}$ & $\begin{array}{l}\mathrm{F} \\
\mathrm{M} \\
\end{array}$ & $\begin{array}{l}\text { Unknown } \\
\text { Unknown } \\
\end{array}$ & $\begin{array}{l}5 / 60 \\
6 / 12 \\
\end{array}$ & $\begin{array}{l}6 / 24 \\
6 / 12 \\
\end{array}$ & $\begin{array}{c}\text { Absent } \\
\text { Absent }\end{array}$ & $\begin{array}{l}\text { Absent } \\
\text { Absent }\end{array}$ & $\begin{array}{l}3 \\
4 \\
\end{array}$ & $\begin{array}{l}2 \cdot 5 \\
4 \\
\end{array}$ & $\begin{array}{l}52 \\
79 \\
\end{array}$ & $\begin{array}{r}64 \\
192 \\
\end{array}$ \\
\hline $\begin{array}{l}38 \\
39\end{array}$ & $\begin{array}{l}12 \\
10\end{array}$ & $\stackrel{\mathrm{M}}{\mathrm{F}}$ & $\begin{array}{l}\text { LMBB } \\
\text { LMBB }\end{array}$ & $\begin{array}{l}6 / 60 \\
6 / 18 \mathrm{pt}\end{array}$ & $\begin{array}{l}6 / 36 \\
6 / 24 \\
\end{array}$ & $\begin{array}{l}\text { Absent } \\
\text { Absent }\end{array}$ & $\begin{array}{l}\text { Absent } \\
\text { Absent }\end{array}$ & $\underline{N}$ & $\begin{array}{l}N \\
-\end{array}$ & $\begin{array}{l}43 \\
24 \\
\end{array}$ & $\begin{array}{l}79 \\
79\end{array}$ \\
\hline 40 & 22 & $\mathrm{~F}$ & X-L (Heterozygote) & $1 / 60$ & $6 / 6$ & $\begin{array}{l}\mathrm{a}-80 \mu \mathrm{v} \\
\mathrm{b}-160 \mu \mathrm{v}\end{array}$ & $\begin{array}{l}\mathrm{a}-80 \mu \mathrm{v} \\
\mathrm{b}-200 \mu \mathrm{v}\end{array}$ & Normal & & 43 & 83 \\
\hline 41 & 8 & F & X-L (Heterozygote) & $\begin{array}{l}2 / 60 \\
\text { myope } \overline{\mathrm{c}}\end{array}$ & $\begin{array}{l}2 / 60 \\
\text { own gls. }\end{array}$ & $\begin{array}{l}\mathrm{a}-60 \\
\mathrm{~b}-120\end{array}$ & - & 2 & $1 \cdot 75$ & 43 & 77 \\
\hline $\begin{array}{l}42 \\
43 \\
44\end{array}$ & $\begin{array}{l}21 \\
32 \\
14\end{array}$ & $\begin{array}{l}\mathrm{F} \\
\mathrm{F} \\
\mathrm{F}\end{array}$ & $\begin{array}{l}\text { X-L (Heterozygote) } \\
\text { AR (Heterozygote) } \\
\text { AR }\end{array}$ & $\begin{array}{l}6 / 4 \\
6 / 5 \\
6 / 4\end{array}$ & $\begin{array}{l}6 / 4 \\
6 / 5 \\
6 / 4\end{array}$ & $\begin{array}{l}\mathrm{N} \\
\mathrm{N} \\
\mathrm{a}-160 \mu \mathrm{v} \\
\mathrm{b}-28 \mu \mathrm{v}\end{array}$ & $\begin{array}{l}\mathrm{N} \\
\mathrm{N}-160 \mu \mathrm{v} \\
\mathrm{b}-240 \mu \mathrm{v}\end{array}$ & $\begin{array}{l}\mathbf{N} \\
\mathbf{N}\end{array}$ & $\begin{array}{l}\mathbf{N} \\
\mathbf{N} \\
\mathbf{N}\end{array}$ & $\begin{array}{l}69 \\
65 \\
53\end{array}$ & $\begin{array}{r}202 \\
90 \\
136\end{array}$ \\
\hline 45 & 35 & $F$ & AR (Heterozygote) & - & - & - & $-\quad 280 \mu$ & - & - & 48 & 127 \\
\hline
\end{tabular}

sixteen it was an autosomal dominant (Cases $13-28$ ), and in three it was sporadic (Cases 29-3I). In four patients the disease was X-linked (Cases 32-35), and the inheritance was unknown in two (Cases 36-37).
In all these patients the fundus appearance was typical of genetically determined retinitis pigmentosa.

There were also two patients with Laurence-MoonBardet-Biedl syndrome (Cases $38-39$ ) and six hetero- 
zygotes from families with recessive disease (Cases 40-45).

Visual loss was due to retinal degeneration in all except Case I, who had lens opacities, and Case 40, who had a right convergent squint with amblyopia. All were patients of Moorfields Eye Hospital.
84 normal volunteers ( 36 males and 48 females) acted as controls; nine were below the age of 20,47 between 20 and 40, 25 between 40 and 60 , and three over 60 years old (Table II).

Table II Vitamin $A$ and beta-carotene plasma level in 84 control subjects

\begin{tabular}{|c|c|c|c|c|c|}
\hline \multicolumn{3}{|c|}{ Males (36) } & \multicolumn{3}{|c|}{ Females (48) } \\
\hline $\begin{array}{l}\text { Age } \\
\text { (yrs) }\end{array}$ & 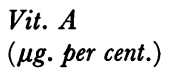 & $\begin{array}{l}\text { beta-carotene } \\
(\mu g . \text { per cent. })\end{array}$ & $\begin{array}{l}\text { Age } \\
\text { (yrs) }\end{array}$ & $\begin{array}{l}\text { Vit. A } \\
(\mu g . \text { per cent. })\end{array}$ & $\begin{array}{l}\text { beta-carotene } \\
(\mu g . \text { per cent. })\end{array}$ \\
\hline 7 & $4^{1}$ & 65 & I 8 & 57 & 112 \\
\hline 14 & $4^{6}$ & 81 & 18 & 74 & 240 \\
\hline 19 & $6 o$ & I 79 & 18 & 27 & 58 \\
\hline $2 \mathrm{I}$ & 66 & 152 & 18 & 71 & 63 \\
\hline 22 & $5^{1}$ & $9^{2}$ & 18 & $6 r$ & 121 \\
\hline 22 & 64 & 223 & 19 & $5^{6}$ & 221 \\
\hline 22 & $5^{I}$ & 54 & 19 & 50 & 75 \\
\hline 23 & $6 o$ & 82 & 20 & 66 & 66 \\
\hline 23 & 69 & I 17 & 20 & 50 & 68 \\
\hline 24 & 46 & 79 & 20 & 46 & 63 \\
\hline 25 & 108 & 136 & 21 & 64 & 85 \\
\hline 26 & 71 & I97 & 21 & 65 & 144 \\
\hline 26 & $6 I$ & 137 & 22 & 38 & 115 \\
\hline 27 & I 17 & 67 & 23 & $6_{5}$ & 134 \\
\hline 27 & 89 & 208 & 23 & 102 & $8 \mathrm{I}$ \\
\hline 28 & 59 & 180 & 24 & 50 & 119 \\
\hline 29 & 78 & 131 & 24 & 46 & 118 \\
\hline 30 & 74 & 105 & 25 & $5^{6}$ & 160 \\
\hline 30 & 64 & 83 & 25 & 107 & 190 \\
\hline $3^{1}$ & $5^{6}$ & $\log$ & 25 & $4^{1}$ & 163 \\
\hline $3^{I}$ & 78 & 187 & 25 & 89 & 92 \\
\hline $3^{1}$ & 66 & 89 & 26 & 93 & 216 \\
\hline 33 & 76 & 152 & 28 & 71 & 102 \\
\hline 34 & 71 & 148 & 29 & 60 & $8_{5}$ \\
\hline 35 & 73 & 212 & 29 & 64 & 187 \\
\hline 39 & 80 & 104 & 33 & $5^{6}$ & 137 \\
\hline 40 & 87 & 32 & 34 & 70 & 103 \\
\hline 42 & 74 & 167 & $3^{8}$ & $3^{1}$ & 132 \\
\hline 42 & 69 & 137 & 39 & $6_{I}$ & 142 \\
\hline 43 & $5^{I}$ & 68 & 39 & 92 & 317 \\
\hline $4^{8}$ & 57 & 97 & 43 & $6_{5}$ & 94 \\
\hline $5^{1}$ & 59 & 63 & 43 & 84 & 165 \\
\hline 54 & 60 & 55 & 44 & 4I & 77 \\
\hline 54 & $9^{2}$ & I 93 & 45 & 46 & 37 \\
\hline 63 & 57 & 57 & 47 & 93 & 130 \\
\hline 65 & 89 & 117 & 47 & 43 & 88 \\
\hline & & & 47 & 46 & $6 \mathrm{I}$ \\
\hline & & & 47 & 64 & I 33 \\
\hline & & & 48 & 53 & II I \\
\hline & & & 49 & 69 & 73 \\
\hline & & & 49 & 48 & 184 \\
\hline & & & 50 & 66 & 144 \\
\hline & & & $5^{I}$ & 87 & I I I \\
\hline & & & 52 & 46 & $13^{8}$ \\
\hline & & & 53 & 93 & 146 \\
\hline & & & 54 & 38 & 95 \\
\hline & & & 58 & 60 & I 19 \\
\hline & & & $6_{5}$ & $6_{5}$ & 248 \\
\hline
\end{tabular}




\section{Methods}

Blood was taken after a 12-hr fast, and the method of Bessey, Lowry, Brock and Lopez (1946), with the modification of Abboud, Osman, and Massoud (1968) for the vitamin $\mathrm{A}$ and beta-carotene assay, was used for sample analysis.

A 4-ml. sample of blood in a lithium sequestrene tube was centrifuged at 3,000 r.p.m. for 15 min., and the plasma transferred into a clean dry plastic tube and frozen immediately for storage. Any sample with haemolysis was discarded.

$0.5 \mathrm{ml}$. plasma was mixed with $0.5 \mathrm{ml}$. alcoholic $\mathrm{KOH}$ (10 per cent) in a $100 \times 12 \mathrm{~mm}$. Pyrex tube and placed in a water bath at $60^{\circ} \mathrm{C}$ for $20 \mathrm{~min}$. After it had cooled to room temperature, I $\mathrm{ml}$. kerosene/xylene mixture was added to each tube and the tube was covered with parafilm. (Kerosene white-Hopkin and. Williams; Xylene-“M \& B. Histologically pure"). Extraction of vitamin $\mathrm{A}$ into the kerosene/xylene layer was achieved by holding the tube at an angle of $45^{\circ}$ against a whirlimixer for $45 \mathrm{sec}$. The tubes were cooled in iced water for $30 \mathrm{~min}$., allowed to return to room temperature, and centrifuged at 3,000 r.p.m. for $15 \mathrm{~min}$.

$0.45 \mathrm{ml}$. of the kerosene/xylene mixture was transferred into quartz microcuvettes for spectrophotometer measurement. The Perkin-Elmer 402 spectrophotometer was used and transmission was measured at $328 \mathrm{~m} \mu$ and $460 \mathrm{~m} \mu$.

The sample was compared with a blank which was prepared in the same way, except that $0.5 \mathrm{ml}$. distilled water was added in the place of plasma.

The mixture was transferred into silica tubes with Teflon stoppers and irradiated with an ultraviolet lamp (Mazda I 25-watt high-pressure mercury lamp $\mathrm{MBW} / \mathrm{U}$ ) for I hr. The solution was returned to clean quartz microcuvettes and their optical density at $328 \mathrm{~m} \mu$ re-measured.

\section{Results}

The levels of blood plasma vitamin $\mathrm{A}$ and betacarotene in the patients are presented in Table I and those of the controls in Table II. On ' $t$ '-test no significant difference was found when the following groups were compared:

Patients and controls (Table III)

Patients and controls of similar ages (Table III)

Patients with autosomal dominant and autosomal recessive disease

Patients with autosomal dominant and X-linked disease Patients with autosomal recessive and $\mathrm{X}$-linked disease Adult male controls and adult female controls

\section{Comment}

This work has failed to find any evidence that systemic vitamin A deficiency plays a role in the pathogenesis of genetically determined isolated retinitis pigmentosa. The failure to demonstrate any difference in plasma levels of vitamin A and beta-carotene between patients with retinitis pigmentosa and controls supports the observations of some workers (Hubbard, 1956; Chatzinoff and others, 1968; Mehra and Khore, 1965), but is at variance with others (Campbell, 1962; Campbell and Tonks, 1962; Campbell and others, 1964; Soliman and others, 1970).

Campbell and Tonks showed persistently low levels of vitamin A in patients with pigmentary retinal degeneration when compared with a control group. Their results for vitamin A levels in both patients (82 i.u./100 ml.) and controls (I 44 i.u./100 ml.)

Table III Pathological "vitamin A"

\begin{tabular}{|c|c|c|c|c|c|c|c|c|}
\hline \multirow[t]{2}{*}{ Comparisons } & \multirow[t]{2}{*}{ Pathological v. normal } & \multicolumn{2}{|c|}{ Patients } & \multicolumn{2}{|c|}{ Controls } & \multirow{2}{*}{$\begin{array}{l}\text { Standard } \\
\text { error }\end{array}$} & \multirow{2}{*}{$\frac{\text { 't'values }}{\text { Calculated }}$} & \multirow[t]{2}{*}{ Significance } \\
\hline & & $n_{1}$ & $y_{1}$ & $n_{2}$ & $y_{2}$ & & & \\
\hline \multirow[t]{3}{*}{ Males } & $\begin{array}{l}\text { Young } \\
\text { (below } 20 \text { yrs) }\end{array}$ & 7 & $6 \mathrm{I} \cdot \mathrm{I} 43$ & 3 & $49^{\circ} 000$ & $7 \cdot 680$ & $I \cdot 58 I$ & $0.05<\mathrm{P}<0.10$ \\
\hline & $\begin{array}{l}\text { Adult } \\
\text { (20 to } 40 \mathrm{yrs} \text { ) }\end{array}$ & 5 & $67 \cdot 800$ & 23 & $70 \cdot 783$ & $8 \cdot 55^{\circ}$ & $0 \cdot 349$ & $0.35<\mathrm{P}<0^{\circ} 4^{\circ}$ \\
\hline & $\begin{array}{l}\text { Adult } \\
\text { (40 to } 60 \mathrm{yrs} \text { ) }\end{array}$ & 3 & $59 \cdot 333$ & 8 & $68 \cdot 625$ & $8 \cdot 500$ & I $\cdot 093$ & $0.15<\mathrm{P}<0.20$ \\
\hline \multirow[t]{3}{*}{ Females } & $\begin{array}{l}\text { Young } \\
\text { (below } 20 \text { yrs) }\end{array}$ & 6 & $47 \cdot 500$ & 6 & $55 \cdot 833$ & $7 \cdot 068$ & $I \cdot I 79$ & $0.10<\mathrm{P}<0.15$ \\
\hline & $\begin{array}{l}\text { Adult } \\
\text { (20 to } 40 \mathrm{yrs} \text { ) }\end{array}$ & I 2 & $60 \cdot 250$ & 24 & $64 \cdot 333$ & $6 \cdot 011$ & 0.679 & $0.25<\mathrm{P}<0.30$ \\
\hline & $\begin{array}{l}\text { Adult } \\
\text { (40 to } 60 \text { yrs) }\end{array}$ & 6 & $63 \cdot 333$ & 17 & $6 I \cdot 294$ & $7 \cdot 835$ & $0 \cdot 260$ & $0.35<\mathrm{P}<0.40$ \\
\hline Total & & 39 & $59 \cdot 821$ & 84 & $64 \cdot 95^{2}$ & $3 \cdot 182$ & $\mathrm{I} \cdot 6 \mathrm{I} 3$ & $0.05<\mathrm{P}<0.10$ \\
\hline
\end{tabular}


were lower than those of other studies. This can probably be accounted for by their use of the relatively insensitive antimony trichloride method which has many disadvantages when compared with spectrophotometry (Abboud and others, 1968). In addition, their control values are those of the Medical Research Council and not their own. Soliman and others (1970) used the same techniques as in this study, and in patients with retinal degeneration they found reduced plasma levels of vitamin A at a low level of significance (I per cent.) and a highly significant reduction in beta-carotene. It is possible that a significant number of their patients had liver disease and no account was taken of dietary factors. Their patients were not restricted to those with genetically determined disease, and some may indeed have had severe vitamin A deficiency and secondary retinal degeneration.

\section{Summary}

Plasma levels of vitamin A and beta-carotene were measured by spectrophotometry in 39 patients with genetically determined retinitis pigmentosa, No difference was found between the levels in these patients and the levels in a control group.

We should like to thank volunteers from Moorfields Eye Hospital and from the Institute of Ophthalmology and traffic wardens who acted as normal controls. We are also grateful to Miss A. M. Rutter for secretarial assistance and to the scientific workshop at the Institute of Ophthalmology for technical assistance.

This work was supported by the Leverhulme Foundation.

\section{References}

abboud, i. A., osman, H. G., and massoud, w. H. (1968) Med. F. Cairo Univ., 36, I BASSEN, I. A., and KORNZWEIG A. L. (I950) Blood, 5, 38I BESSEY, O. A., LOWRY, O. H., BROCK, M. J., and LOPEZ, J. A. (1946) 7. biol. Chem., 166, I 77 Billings, J. J., o'callaghan, J., and o'dAy, k. (1957) Trans. ophthal. Soc. Aust., 17, I3 I CAMPBELL, D. A. (1962) Trans. ophthal. Soc. U.K., 82, 667 , HARRISON, R., and TONKS, E. L. (1964) Exp. Eye Res., 3, 412 and TONKs, E. L. (1962) Brit. F. Ophthal., 46, I5 1

Ghatzinoff, A. B., Nelson, E., Stahl, N., and Glahane, A. (1968) Arch. Ophthal. (Chicago), 80, 4 I 7 COGAN, D. G. (1950) Trans. Amer. Acad. Ophthal. Otolaryng., 54, 629

FELLS, P., and BORS, F. (1969) Trans. ophthal. Soc. U.K., 89, 22 I

and (1971) Brit. F. Ophthal., 55, 210

FLYNN, P., and AIRD, R. B. (1965) F. neurol. Sci., 2, I6I FRIEDE, R. (1952) Klin. Mbl. Augenheilk., 120, 605

hubBard, R. J. (1956) f. gen. Physiol., 39, 935 JAMPEL, R. S., OKAZAKI, H., and BERNSTEIN, H. (196I) Arch. Ophthal. (Chicago), 66, 247 KEARNS, T. P. (1965) Trans. Amer. ophthal. Soc., 63, 559

LAURENCE, J. z., and MOON, R. c. (1866) Ophthal. Rev., 2, 32

LEVine, J. (1933) Arch. Ophthal. (Chicago), 9, 453

LEVY, I. S. (1970) Trans. ophthal. Soc. U.K., 90, I8I

LLOYD, J. K. (I968) Arch. Dis. Childh., 43, 393

MCLAREN, D. s. (1963) "Malnutrition and the Eye". Academic Press, New York

MEHRA, K. s., and KhARE, B. B. (1965) Orient. Arch. Ophthal., 3, 8o

NORDEN, A., and STIGMAR, G. (1969) Acta ophthal. (Kbh), 47, 716

Refsum, S., SAlmonsen, L., and SKATVedt, M. (I949) F. Pediat., 35, 335

RODGER, F. C. (1966) Trans. ophthal. Soc. U.K., 86, I 77

SHEARER, A. c. I. (1964) Exp. Eye Res., 3, 427

SOliman, A. M., ABBOUd, I. A., OSMAN, H. G., and massoud, W. H. (1970) "XXI Concilium Ophthalmologicum

Mexico, 1970", ed. M. P. Solanes, pt. 2, p. I806. Excerpta Medica, Amsterdam

SPERLiNG, M. A., hiles, D. A., and keNNerdell, J. s. (1972) Amer. F. Ophthal., 73, 342

TOWN, A. E. (195I) “Ophthalmology", p. 443. Kimpton, London

WEINER, L. P., KONisMark, B. W., Stoll, J., and magladery, J. W. (1967) Arch. Neurol., I6, 364

winkelman, N. w. (1932) Arch. Neurol. Psychiat., 27, I 